\title{
Dietary energy compensation in response to reduced sugar diet in non-obese men and women
}

\author{
O. Markey ${ }^{1,2}$ and J. A. Lovegrove ${ }^{1,2}$ \\ ${ }^{1}$ Hugh Sinclair Unit of Human Nutrition and ${ }^{2}$ Institute for Cardiovascular and Metabolic Research (ICMR), \\ Department of Food and Nutritional Sciences, University of Reading, Reading, RG6 6AP, UK
}

Consumption of reformulated sugar-containing processed products has been recommended as a strategy for reducing non-milk extrinsic sugars (NMES) to no more than $10 \%$ of total energy intake (EI) and as a weight reducing strategy in today's obesity epidemic ${ }^{(1,2)}$. It is therefore important to determine the impact of sugar reformulated food consumption on energy balance dynamics.

The REFORM study (ClinicalTrials.gov NCT01645995) examined the impact of an 8-week sugar reformulated product exchange on EI, energy expenditure (EE) and energy balance (EB), in a double-blind, randomised, controlled, crossover design. Forty five healthy, non-dieting, non-obese volunteers (14 male, 31 female; age: 32.0 (SD 9.8) years; BMI: 23.5 (SD 3.0) kg/m²) were randomly assigned to consume either regular sugar or sugar-reformulated food and drink products including confectionary, pasta sauces, baked beans, muesli, condiments and sugar-sweetened beverages for a $56 \mathrm{~d}$ period, with a $28 \mathrm{~d}$ washout period. The minimum target difference in dietary NMES intake between the regular and reformulated product exchange was $38 \mathrm{~g} / \mathrm{d}(152 \mathrm{kcal})$. Weighed food diaries (4 d) and accelerometer data (7 d) were used to assess EI, EE and EB at baseline and following each dietary exchange period.

There was no significant effect of the intervention on body weight, EI, EE or EB. Significant time $\mathrm{x}$ treatment interactions were evident for carbohydrate $(\mathrm{CHO}$; g and $\% \mathrm{EI} ; P<0.000)$, total sugar intake (g and \% EI; $P<0.000)$ and fat $\% \mathrm{EI} ; P<0.001$ ). Significantly higher $\mathrm{CHO}$, total sugar and lower fat as a percentage of EI were consumed following the regular sugar treatment. Body weight at the end of the reformulated dietary exchange period was predicted to be 2.5 (SE 0.001) $\mathrm{kg}$ lower using the National Institute of Diabetes and Digestive and Kidney (NIDDK) body weight simulator ${ }^{(3)}$, compared to the observed body weight. The predicted body weight accounted for $98.8 \%$ of the variance in the observed body weight, suggesting that the volunteers' energy compensated.

Long-term sugar reformulated product exchange resulted in energy compensation, when consumed as part of an habitual diet, partly through increased voluntary fat intake. Furthermore, body weight was predicted to be lower (using the NIDDK model) than observed, following the reformulated dietary exchange period, supporting the hypothesis of dietary energy compensation. In conclusion consumption of reformulated-sugar products as part of a blinded dietary exchange, resulted in a significant reduction in sugar intake, but had no effect on energy balance or body weight due to dietary energy compensation in non-obese men and women. Future work is required to determine if these findings have relevance to energy compensation in obesity and if consumed without blinding.

This research was supported by Sugar Nutrition UK

1. Department of Health, 1991, Dietary Reference Values for Food Energy and Nutrients for the United Kingdom. Vol. 41: Report on Health and Social Subjects. London: Her Majesty's Stationery Office.

2. van Raaij J, Hendriksen M, Verhagen H (2009) Public Health Nutr 12, 325-330.

3. Hall et al. 2011, Lancet 378, 826-837. 